\title{
Virtual Instruction On Demand for Educational Orientation of Graduate Medical Education Trainees During The COVID-19 Pandemic
}

\section{Scott Holliday}

The Ohio State University Wexner Medical Center

Nasir Hussain

The Ohio State University Wexner Medical Center

Matthew Lang

The Ohio State University Wexner Medical Center

Coranita Burt

The Ohio State University Wexner Medical Center

Amber Clevenger

The Ohio State University Wexner Medical Center

Jeffery Barbee

The Ohio State University College of Medicine

Amanda Start

The Ohio State University College of Medicine

Juan Fiorda-Diaz

The Ohio State University Wexner Medical Center

Daniel Clinchot

The Ohio State University College of Medicine

Michael Essandoh ( $\square$ michael.essandoh@osumc.edu )

The Ohio State University Wexner Medical Center

\section{Research Article}

Keywords: COVID-19, Resident physician onboarding, Virtual orientation, Graduate Medical Education.

Posted Date: September 7th, 2021

DOI: https://doi.org/10.21203/rs.3.rs-737910/v1

License: (c) (1) This work is licensed under a Creative Commons Attribution 4.0 International License.

Read Full License 


\section{Abstract}

Background: Graduate medical education (GME) orientation is traditionally an in-person endeavor. The COVID-19 pandemic has prompted virtual approaches to trainee onboarding to reduce viral transmission; however, virtual orientation has not been well-described in GME. This study assesses the effectiveness of virtual orientation of GME trainees using data from an electronic survey.

Objectives: We aimed to determine the usefulness of virtual instruction on demand for GME trainee onboarding at a large sponsoring institution.

Methods: We conducted a retrospective analysis of de-identified electronic survey responses by residents and fellows who underwent GME orientation at our institution from June to August 2020. The primary outcome was to determine the effectiveness of virtual GME orientation for onboarding, and secondary outcomes included identifying barriers to implementation and weaknesses associated with virtual GME orientation. We conducted orientation sessions for the incoming residents and fellows using CarmenCanvas, our institutional learning management system (LMS).

Results: 272 of 337 incoming trainees completed the virtual GME orientation survey. $97 \%$ of respondents reported that the contents of the orientation modules would help them perform their duties. $79 \%$ of trainees rated the overall quality as very good or good, $91 \%$ responded that virtual orientation provided effective learning, $94 \%$ reported accessing the course content easily, $92 \%$ reported easy navigation of the modules, $91 \%$ described it as well-organized, and $87 \%$ said that the modules supported their learning.

Conclusions: Virtual instruction on demand is a safe, effective, and practical approach to resident and fellow onboarding into a GME sponsoring institution in the COVID-19 pandemic era.

\section{Background}

The virulent nature of the severe acute respiratory syndrome coronavirus 2 (SARS-CoV-2) has spurred a need to create and implement guidelines to reduce human-to-human transmission. ${ }^{1,2}$ As developed by the Centers for Disease Control and Prevention (CDC) and the World Health Organization (WHO), these guidelines have several recommendations, which include safe social distancing of at least six feet, reduced in-person human contact, frequent hand washing, respiratory etiquette, and mask-wearing. ${ }^{2}$

Residency and fellowship programs have faced significant academic and operational challenges in the setting of this global misfortune. ${ }^{3-10}$ Schwartz et al. ${ }^{4}$ recently summarized a clear pathway to preserve resident education and training while prioritizing patient care. ${ }^{4}$ Likewise, medical centers across the United States have pivoted in this direction by establishing protocols to treat COVID-19 infections while preventing transmission to other patients and healthcare workers. These protocols, which enforce social distancing, have further affected the integration of new trainees into Graduate Medical Education (GME) sponsoring hospitals. 
Historically, onboarding trainees into GME sponsoring hospitals has been an in-person activity ${ }^{11-13}$, but the rise of COVID-19 and the requirement for safe social distancing makes this challenging. Sponsoring institutions have already pivoted towards the virtual integration of trainees; however, there are limited best practices, guidelines, recommendations, or institutional outcomes data to help GME personnel integrate trainees into their institution during this unprecedented era.

Thus, this cross-sectional study investigates the effectiveness of Virtual Instruction on Demand for Educational Orientation (VIDEO) of GME trainees during the COVID-19 pandemic using an electronic survey developed by our institution. Specifically, using this survey, we sought to examine the i) satisfaction of residents for remote GME training and ii) better understand the barriers or challenges of implementing such a tool for future GME orientations.

\section{Methods}

Per our standard protocol for in-person GME orientation, we conducted a cross-sectional survey of residents and fellow trainees who underwent virtual GME orientation at our institution between June 1 , 2020, and August 31, 2020. We attained institutional review board approval (IRB \#2020E0868) to perform a retrospective analysis of the GME virtual orientation process. Considering the retrospective nature of the study, informed consent was waived for all subjects, and all methods were carried out under our institutional guidelines and regulations for such studies.

\section{Sampling Frame}

We intended our survey to be administered to both incoming residents and fellows. Our institution has representation from a wide variety of training programs, including 75 accreditation council of graduate medical education (ACGME) residencies and fellowships; and 80 non-ACGME accredited programs. Our programs span the gamut of specialty and subspecialty, including medical, surgical, hospital-based specialties, dental, podiatry, and psychology.

\section{Orientation Process}

We conducted orientation sessions for the incoming residents and fellows using CarmenCanvas, our institution's version of the Canvas ${ }^{\circledR}$ Learning Management System (LMS). The orientation sessions comprised modules that covered topics deemed essential for incoming trainee onboarding. Selected faculty members and GME staff created the modules and subsequently uploaded them onto CarmenCanvas. These modules centered on the themes considered critical for trainee integration, such as point-of-care ultrasound, effective handoffs, line, and tube placement, epidemiology and COVID-19, patient safety, medical information management, professionalism, and seeing the virtual patient. A full description of the goals and objectives of each module can be viewed in Appendix A. It was obligatory for trainees matriculating into residency or fellowship programs at our Medical Center to complete the orientation modules over ten days. Upon completing the modules, the trainees completed a questionnaire developed to assess the training modules' usefulness, although this was not mandatory. 


\section{Survey Development}

The GME Office and our College of Medicine's Office of Curriculum and Scholarship collaborated to create the survey to assess trainee satisfaction, challenges, and barriers to the online GME onboarding sessions. Items on the questionnaire covered seven broad domains, which included: usefulness of the orientation materials to performance of trainee duties, overall quality of the GME orientation, the efficacy of the virtual orientation process for trainee learning, ease of access, and ease of navigation of the CarmenCanvas orientation material, organization of the orientation process and whether the modules supported trainee learning. The survey comprised only closed-ended questions with categorical responses on a Likert scale. Specifically, responses ranged from: "rarely" to "frequently" for the projected use of the orientation content (4-point Likert scale); "very poor" to "very good" when rating the overall quality of the virtual GME orientation (5-point Likert scale); "very ineffective" to "very effective" when ranking individual learning (6-point Likert scale). The ease of access and navigation, level of organization, and the learning support provided by the orientation material, were rated from "strongly disagree" to "strongly agree" (5-point Likert scale). A complete list of the questions and response choices can be viewed in Appendix B.

\section{Survey Administration}

We invited both incoming resident trainees and fellows to complete the VIDEO study survey - the only prerequisite was completing all modules and submitting all assignments before taking the survey. We embedded the survey into the LMS. Participants received up to five email reminders to complete the survey. The orientation modules were available for trainee access over ten days, and the trainees could meet their orientation expectations on their own timeline. Instructions and a syllabus were sent to all trainees electronically to help them navigate the system and find the correct course. The CarmenCanvas platform has a built-in communication system that facilitates direct communication between trainees and GME personnel, and so technical difficulties could be solved rapidly.

\section{Statistical Analysis}

Data recording was carried out on CarmenCanvas. Once completed, raw data was de-identified and distilled into tables, graphs, and figures using a Microsoft Excel Worksheet (2016). All survey respondents' descriptive statistics, including gender, racial ethnicity, and medical specialty, were analyzed. Likewise, we analyzed the frequency of responses for each question on the whole. Finally, we performed a multiple linear regression analysis to identify factors that may predict satisfaction with virtual GME, including gender and racial ethnicity. All analyses were 2-tailed, and we considered the results to be significant at $p<0.05$.

\section{Results}

We invited 353 trainees enrolled in the virtual GME orientation to complete the online survey via CarmenCanvas. Of these, 272 trainees, including 191 residents and 81 fellows, responded to the 
questionnaire for a survey response rate of $77 \%$. Completing the survey was not mandatory, and the survey was available for completion after the trainees completed their orientation modules.

\section{Characteristics of Trainee Respondents}

Of the 272 trainees that completed the survey, $54 \%(n=147)$ were male, and $46 \%(n=125)$ were female. Most of the trainees were incoming resident physicians at 70\% $(n=191)$, with fellows only comprising $30 \%(n=81)$ of survey respondents. Regarding ethnicity, most respondents were white at $66 \%(n=181)$; in contrast, $19 \%(n=52)$ were Asian, 5\% $(n=14)$ were Black or African American, and 4\% $(n=10)$ were Hispanic or Latino. As expected, participation was best represented in the survey by our largest program, Internal Medicine, at 33\% ( $n=89)$. In contrast, smaller programs such as Neurological Surgery and Emergency Medicine / Internal Medicine were the least represented at 1\% $(n=2)$ each (Table 1). 
Table 1

Demographic characteristics.

\begin{tabular}{|ll|}
\hline Demographic & No. (\%) \\
\hline Gender & $147(54 \%)$ \\
Fale & $125(46 \%)$ \\
\hline Ethic Origin & \\
Asian & $52(19 \%)$ \\
White & $181(66 \%)$ \\
Black or African American & $14(5 \%)$ \\
Hispanic or Latino & $10(4 \%)$ \\
Other & $15(6 \%)$ \\
Trainee Type & $191(70 \%)$ \\
Resident & $81(30 \%)$ \\
Fellow & \\
\hline
\end{tabular}




\begin{tabular}{|ll|}
\hline Demographic & No. (\%) \\
\hline Specialty of Training & $9(3 \%)$ \\
Internal Medicine / Pediatrics & $13(5 \%)$ \\
Psychiatry & $21(8 \%)$ \\
Emergency Medicine & $6(2 \%)$ \\
Plastic Surgery & $20(7 \%)$ \\
Anesthesiology & $9(3 \%)$ \\
Orthopedic Surgery & $13(5 \%)$ \\
Family Medicine & $89(33 \%)$ \\
Internal Medicine & $15(6 \%)$ \\
Radiology & $14(5 \%)$ \\
Obstetrics and Gynecology & $7(3 \%)$ \\
Otolaryngology & $17(6 \%)$ \\
Surgery & $9(3 \%)$ \\
Neurology & $14(5 \%)$ \\
Dentistry & $3(1 \%)$ \\
Urology & $4(2 \%)$ \\
PM\&R & $2(1 \%)$ \\
Emergency Medicine / Internal Medicine & $2(1 \%)$ \\
\hline Neurological Surgery & $5(2 \%)$ \\
\hline Pathology & \\
\hline
\end{tabular}

\section{Outcomes of the Survey}

\section{Usefulness of Orientation Content for Performance of Trainee Duties}

At $51 \%(n=140)$, most respondents reported they would frequently use the content from the orientation to perform clinical duties. Only a small proportion, $3 \%(n=8)$, felt it would minimally affect their clinical duties' performance (Table 2). 
Table 2

Descriptive of survey responses.

\begin{tabular}{|c|c|}
\hline Question & No. (\%) \\
\hline $\begin{array}{l}\text { How frequently will you use the content from this orientation to perform your duties at } \\
\text { OSUWMC? }\end{array}$ & $\begin{array}{l}140 \\
(51 \%)\end{array}$ \\
\hline Frequently & $38(14 \%)$ \\
\hline Occasionally & $86(3 \%)$ \\
\hline Sometimes & $8(3 \%)$ \\
\hline \multicolumn{2}{|l|}{ Rarely } \\
\hline How would you rate the overall quality of your graduate medical education orientation? & $78(29 \%)$ \\
\hline Very good & $\begin{array}{l}132 \\
(48 \%)\end{array}$ \\
\hline Good & $58(21 \%)$ \\
\hline Acceptable & $3(1 \%)$ \\
\hline Poor & $1(1 \%)$ \\
\hline Very poor & \\
\hline How effective or ineffective was this virtual orientation for your learning? & $47(17 \%)$ \\
\hline Very effective & $\begin{array}{l}118 \\
(43 \%)\end{array}$ \\
\hline Effective & $82(30 \%)$ \\
\hline Somewhat effective & $14(5 \%)$ \\
\hline Somewhat ineffective & $10(4 \%)$ \\
\hline Ineffective & $1(1 \%)$ \\
\hline Very ineffective & \\
\hline The Carmen course was easy to access. & $\begin{array}{l}137 \\
(50 \%)\end{array}$ \\
\hline Strongly agree & $\begin{array}{l}117 \\
(43 \%)\end{array}$ \\
\hline Neutral & $15(6 \%)$ \\
\hline Disagree & $2(1 \%)$ \\
\hline Strongly disagree & $1(1 \%)$ \\
\hline
\end{tabular}




\begin{tabular}{|ll|}
\hline Question & No. (\%) \\
\hline The Carmen course was easy to navigate. & 125 \\
Strongly agree & $(46 \%)$ \\
Agree & 125 \\
Neutral & $(46 \%)$ \\
Disagree & $17(6 \%)$ \\
Strongly disagree & $5(2 \%)$ \\
\hline The orientation was well organized. & $0(0 \%)$ \\
Strongly agree & 102 \\
Agree & $(38 \%)$ \\
Neutral & 144 \\
Disagree & $(53 \%)$ \\
Strongly disagree & $21(8 \%)$ \\
\hline The modules supported my learning & $5(2 \%)$ \\
Strongly agree & $0(0 \%)$ \\
Agree & $67(25 \%)$ \\
Neutral & 168 \\
Disagree & $(62 \%)$ \\
Strongly disagree & $27(10 \%)$ \\
\hline OVerall alualtV & $9(3 \%)$ \\
\hline
\end{tabular}

\section{Overall quality of the GME orientation}

The online GME orientation modules were described to be of "good" or "very good" quality by $77 \%(\mathrm{n}=$ $210)$ of respondents. Only $2 \%(n=4)$ of respondents felt that the quality was poor or very poor (Table 2$)$.

\section{Efficacy and support of the virtual orientation for trainee learning}

Another important parameter on the survey was the effectiveness of our novel approach to trainee onboarding for trainee learning. 47 trainees $(17 \%)$ reported that the remote presentation of GME orientation was very effective for learning the orientation materials. Significantly, few trainees preferred an in-person approach, which was not achievable because of the pandemic. Specifically, only $5 \%(n=11)$ of respondents felt that the virtual orientation was ineffective or very ineffective (Table 2).

\section{Ease of access of CarmenCanvas}


The trainees reported accessing the modules quickly during the orientation period $-93 \%(n=254)$ said that the modules were straightforwardly accessed (Table 2).

\section{Ease of navigation of CarmenCanvas}

The survey results showed that the questionnaire's navigation was easy, and trainees could complete it without difficulty, at $92 \%(n=250)$. A few trainees shared some challenges, but this was a small proportion of the respondents at $2 \%(n=5)$ (Table 2$)$.

\section{Organization of the orientation process}

Regarding accessibility, the trainees received an email with detailed instructions for accessing and completing the orientation materials. They also received information on who to contact if they had any difficulties with the GME orientation process. As a result, $91 \%(n=246)$ of respondents reported that the virtual orientation was well organized, with no identifiable barriers to survey access or completion (Table 2).

\section{Support of Learning}

At $87 \%(n=235)$, most respondents felt that the online GME modules supported their learning. Only $4 \%$ ( = 10) of respondents thought the modules did not benefit their training (Table 2).

\section{Impact of Race and Gender on survey responses}

We stratified survey responses according to gender (male and female), ethnicity (Asian, White, Block or African American, Hispanic or Latino, or "other"), and medical sub-specialty. Overall, multiple linear regression did not reveal significant differences in all survey questions based on gender, ethnicity, or medical sub-specialty.

\section{Discussion}

Technology has transformed educational programs and has created an inexhaustible source of information and communication accessible to anyone worldwide. Leveraging technology for trainee orientation in the COVID-19 pandemic is alluring; however, published literature describing the safety and effectiveness of virtual education for resident and fellow integration into a sponsoring program is limited. 14 Our manuscript highlights the value of this unique approach to learner onboarding.

In a recent report, Schwartz et al. ${ }^{4}$ describe their orthopedic residency program's education and training strategies during the current pandemic. ${ }^{4}$ They divided the residents into two cycling groups: the "active duty" and the "working remotely" groups, with the former maintaining in-person surgical training. The "working remotely" group only took part in virtual educational activities. The two teams transitioned every two weeks to reduce COVID-19 exposure. Although this approach was successful and preserved the resident workforce, adopting this strategy in GME orientation may not be practical considering the large number of learners. 
Resident and fellow trainees entering our Medical Center's GME training programs participate in an institutional GME orientation to acclimate them to the unfamiliar learning environment. Before the COVID19 pandemic, we performed GME onboarding in an in-person, small-group station-based, active learning approach to critical principles and procedures applicable to all specialties in GME. However, the COVID-19 pandemic prevented our standard in-person orientation process because of the absolute need for social distancing. As a result, we creatively pivoted to a virtual GME orientation process in 2020.

After considerable planning, educational/training modules were designed using similar content from our traditional in-person approach and uploaded into our LMS. We maintained the in-person curriculum, even around procedural safety techniques and understanding the clinical learning environment, yet successfully transitioned to an online delivery platform to comply with the CDC and WHO recommendations to reduce the risk of COVID-19 transmission. Our analysis of the trainee feedback data suggests that virtual GME orientation using CarmenCanvas is practical and provides residents and fellows with the information needed to adjust to their new clinical learning environment. The trainees overwhelmingly appreciated the ability to complete the modules remotely, described the contents as beneficial to their education and training, and had little difficulty accessing and navigating relevant content. They appreciated the virtual orientation organization and the flexibility that the virtual platform provided.

\section{Lessons Learned}

Our strategy did not require changing our orientation materials' content, but only the delivery platform. We did not encounter any barriers to implementing virtual orientation. The virtual CarmenCanvas platform enabled the provision of GME orientation to late-arriving residents and eliminated the scheduling of makeup lectures as occurs with the in-person format. Based on survey responses, we found this change to be very successful. Of note, specific modules that were traditionally hands-on (i.e., invasive procedures) were presented as video recordings to emphasize the procedures' key aspects.

The VIDEO study helped us identify areas for improvement using this novel virtual orientation format using trainee respondents' comments. A few trainees expressed that despite the lecture format's informative nature (PowerPoint slides with voiceover recordings), an interactive design such as videoconferencing would be more helpful, especially for questions and answers, and we intend to use this approach in the future. Second, we also had variability in the modules' duration. The respondents requested standardization of the length of modules (the majority preferred shorter, concise lectures of $\sim$ 30 minutes duration) to improve trainee engagement.

\section{Study Limitations}

Our study has limitations inherent to its cross-sectional nature. However, the data presented were prospectively collected in a large GME sponsoring institution and provides insight into virtual onboarding. Another limitation was the lack of hands-on experiential training for specific procedures, such as airway management and invasive vascular access. We should note that these procedural trainings were re- 
incorporated using small group active learning sessions in the subsequent weeks. Using a virtual format may not provide trainees with the best skills to perform such procedures. Consideration should be given to a limited group session for trainees who need such expertise for patient care using physical distancing, wearing personal protection equipment, and strict hand hygiene.

\section{Conclusions}

Our VIDEO study results show that virtual instruction on demand is a safe and effective method for trainee integration into GME sponsoring hospitals. Besides, it is a potentially cost-effective approach from a training and education perspective, with minimal implementation barriers. Undoubtedly, the inability to assemble residents has been a significant roadblock, but our ability to pivot to a virtual platform made orientation feasible and safe. Virtual onboarding of GME trainees must be performed thoughtfully, and eventually, this may become standard practice as infectious diseases such as COVID-19 remain prevalent. GME programs need to share their unique onboarding processes as we navigate this unprecedented time, to facilitate the creation of best practices for virtual trainee integration into GME sponsoring institutions.

\section{Abbreviations}

GME

Graduate medical education

SARS-CoV-2

Severe Acute Respiratory Syndrome Corona virus 2

$\mathrm{CDC}$

Centers for Disease Control and Prevention

WHO

World Health Organization

VIDEO

Virtual Instruction on Demand for Educational Orientation

LMS

Learning Management System

\section{Declarations}

\section{Ethics Approval and Consent to Participate}

This is a retrospective review and so informed consent was not required by our internal IRB, (IRB \#2020E0868).

\section{Consent for Publication}

Consent for publication was waived by our IRB. 
Availability of Data and Material

Yes, all data and material included in this manuscript are available for review upon request. Data can be requested from Michael Essandoh for review.

\section{Competing Interests}

None

\section{Funding}

None

\section{Authors Contributions}

$\mathrm{SH}, \mathrm{ME}, \mathrm{NH}, \mathrm{DC}$, and $\mathrm{ML}$ developed the idea and created the study; $\mathrm{SH}, \mathrm{NH}, \mathrm{DC}$, and ME wrote the main manuscript; $\mathrm{ML}, \mathrm{CB}$, and $\mathrm{AC}$ performed data collection; JB, AS, JFD, $\mathrm{NH}$, and ME performed data analysis; All authors reviewed the manuscript before submission for publication.

\section{Acknowledgments}

None

\section{References}

1. Poland GA. SARS-CoV-2: a time for clear and immediate action. The Lancet Infectious Diseases. 20.5 (2020): 531-532..

2. Organization WH. Infection prevention and control during health care when COVID-19 is suspected: interim guidance, June 29 2020. World Health Organization. 2020;No. WHO/2019nCoV/IPC/2020.4(2020).

3. Iyer P, Aziz K, Ojcius DM. Impact of COVID-19 on dental education in the United States. Journal of Dental Education. 2020;84(6):718-722.

4. Schwartz AM, Wilson JM, Boden SD, Moore Jr TJ, Bradbury Jr TL, Fletcher ND. Managing resident workforce and education during the COVID-19 pandemic: evolving strategies and lessons learned. JBJS Open Access. 2020;5(2):e0045.

5. Karl H. Pang, Diego M. Carrion, Juan Gomez Rivas, Guglielmo Mantica, Angelika Mattigk, Benjamin Pradere, et al. The impact of COVID-19 on European health care and urology trainees. European Urology. 2020 July 1.

6. Daodu O, Panda N, Lopushinsky S, Varghese Jr TK, Brindle M. COVID-19-considerations and implications for surgical learners. Annals of surgery. 2020;272(1):e22-e23.

7. Fuller S, Vaporciyan A, Dearani JA, Stulak JM, Romano JC. COVID-19 disruption in cardiothoracic surgical training: an opportunity to enhance education. The Annals of Thoracic Surgery. 2020 
Nov;110(5):1443.

8. Potts JR. Residency and fellowship program accreditation: effects of the novel coronavirus (COVID19) pandemic. Journal of the American College of Surgeons. 2020;230(6):1094-1097.

9. Zhi Ven Fong, Motaz Qadan, Ross McKinney Jr, Cornelia L. Griggs, Paresh C. Shah, Jo Buyske, et al.

Practical implications of novel coronavirus COVID-19 on hospital operations, board certification, and medical education in surgery in the USA. Journal of Gastrointestinal Surgery. 24(6), pp.1232-1236.

10. Chong A, Kagetsu NJ, Yen A, Cooke EA. Radiology residency preparedness and response to the COVID-19 pandemic. Academic radiology, 27(6), pp.856-861

11. Chen LY, McDonald JA, Pratt DD, Wisener KM, Jarvis-Selinger S. "Residents' views of the role of classroom-based learning in graduate medical education through the lens of academic half days. Academic Medicine. 2015;90(4):532-538.

12. William Wood, Jonathan McCollum, Promil Kukreja, Imelda L. Vetter, Charity J. Morgan, Ana Hossein Zadeh Maleki, et al. Graduate medical education scholarly activities initiatives: a systematic review and meta-analysis. BMC medical education. 2018;18(1):318.

13. Education ACGME. ACGME common program requirements. https://www.acgme.org/portals/0/pfassets/programrequirements/cprs_2017-07-01.pdf 2017; effective: July 1, 2017. Accessed September 01, 2020.

14. Moffett J. Twelve tips for "''flipping"'' the classroom. Medical teacher. 2015;37(4):331-336.

\section{Figures}




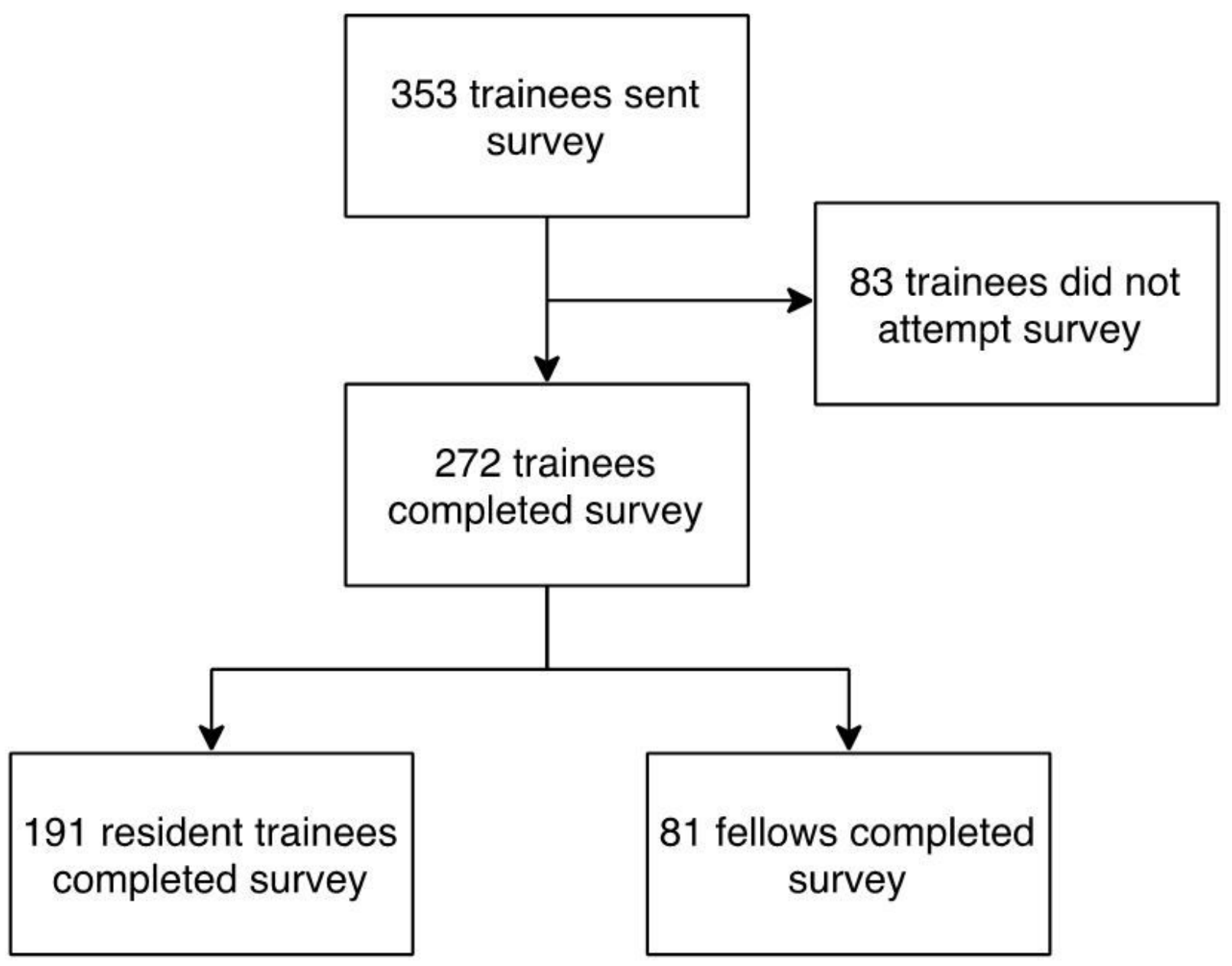

Figure 1

Study flow diagram.

\section{Supplementary Files}

This is a list of supplementary files associated with this preprint. Click to download.

- AppendixA1.docx

- AppendixB.docx 